\title{
New Insight into the Graphene Based Films Prepared from Carbon Fibers
}

\author{
Yanxiang Wang", Wenxin Fan, Guoli Wang, Minxia Ji \\ Carbon Fiber Engineering Research Center of Shandong Province, College of Materials Science and Engineering, Shandong Univer- \\ sity, Jinan, China. \\ Email: wyx079@sdu.edu.cn
}

Received October $13^{\text {th }}, 2010$; revised January $10^{\text {th }}, 2011$; accepted May $21^{\text {st }}, 2011$.

\begin{abstract}
In this work, ultrathin sections from longitudinal polyacrylonitrile (PAN) based T700 and T300 carbon fibers were prepared by ultramicrotomy, a promising graphene based thin films were developed in one step at ambient temperature. It is investigated that the network-graphene planes composed with carbon atoms are partly straight and partly twisted in the thin films prepared from T700 carbon fibers, the distance between the carbon atoms of network-graphene plane decreases, the order design of graphene in the films prepared from 7700 carbon fibers is denser and its arrangement shows a preferred orientation along the drawing direction, its consistency of the neighboring graphene based planes is better, moreover, the relative content of the forming $S P^{2}$-hybridized orbit of carbon atoms in the films prepared from T700 carbon fibers is higher, in the other words, the fact of the graphene based film prepared from carbon fibers without having the characteristic of skin-core structure has been verified.
\end{abstract}

Keywords: Carbon Materials, Microstructure, Graphene, Thin Films

\section{Introduction}

In recent years, carbon materials have gained an increasing attraction in more and more fields owing to its extensive application ranges and extreme-potential perspectives, which are rapidly becoming the global research hot spot [1-3]. The preparation methods of graphene based carbon materials mainly included mechanical exfoliating [4-6], oxidation reduction process of graphite [7-9], solution method [10-13], and chemical method [14-19]. But the yield of obtained graphene based on the mechanical exfoliating method was lower. Although large scale uniform graphene based films could be synthesized by the oxidation reduction process of graphite, the electronic structure and crystalline integrity of graphene were heavily damaged by the strong oxidizer. The graphene based thin-film semiconductor with the large area could be produced by the chemical method, but its application was limited by the immature technology. As we know, the carbon atoms of graphene are bonded by the covalent bond [20]. During research of polyacrylonitrile (PAN) based carbon fibers, some valuable research achievement have been obtained [21,22]: the high strength of carbon fibers are based on the preferred orientation of highly extended crystalline structure along fiber axis and high bond energy among the interfacial layers. We also verified the Ribbon model put forward by Perret [23] and the two-dimensional model proposed by Bennet [24]: the skins of PAN based carbon fibers have a remarkable anisotropy [25], the elementary unit of stripe model is made up of the $s p^{2}$-hybridized carbon atoms [20]. The examination not only verifies some results presented previously but also gives some new features of PAN based carbon fibers that have not proposed in literature. To the best of our knowledge, it is the first time to report a detailed investigation on the novel cutting technique that the graphene based films could be prepared from PAN based carbon fibers.

\section{Experimental Section}

\subsection{Samples Prepared}

PAN based carbon fibers were charged with the electricity for 5 minutes, the samples were immersed into the acetone liquid for $12 \mathrm{~h}$. The solution of epoxy resin prepared was poured into the rubber mould. A tow of PAN fibers soaked was cut and embedded in epoxy resin solution. The mould was dried into the oven at $60^{\circ} \mathrm{C}$. After solidification, ultrathin sections of samples from longitu- 
dinal parts were cut respectively on the Reichert-Jung Ultracut $\mathrm{E}$ ultramicrotome using a diamond knife. The ultrathin sections of samples collected from the distilled water and were dried to test.

\subsection{Characterization}

The specimens were examined using a Philips Tecnai 20U-Twin high-resolution transmission electron microscopy (HRTEM) at an accelerating voltage of $200 \mathrm{kV}$. A Rigaku X-ray diffractometer (D/MAX-rA, Japan) with $\mathrm{Ni}$-filtered $\mathrm{CuK}_{\alpha}$ radiation as the source was used to study the wide angle at $40 \mathrm{KV}$ and $60 \mathrm{~mA}$, the scanning speed was $6 \mathrm{deg} / \mathrm{min}$, scanning step was $0.02 \mathrm{deg}$. The Raman spectrum were taken with Raman scope of LRSLabRam-1B from Jobinyvon with $\mathrm{He}-\mathrm{Ne}$ gas laser as the light source, its laser power was $4.3 \mathrm{~mW}$, the excitation laser was $632.8 \mathrm{~nm}$.

\section{Results and Discussion}

\subsection{HRTEM Analysis of Graphene Based Films}

HRTEM images of ultrathin sections prepared from T700 carbon fibers are investigated in Figure 1, it has been found that such graphene based films have the better orientation structures and more graphene layers which regularly piled up and highly oriented, its consistency of the adjacent graphene based planes is better, several graphene layers are closely arranged in the ordered orientation texture. The length of each graphene layer in such films is longer which was about $7 \mathrm{~nm}$ on the average, the stack height $\left(L_{c}\right)$ and the width $\left(L_{a}\right)$ of such graphenes are obviously seen in the range of $1.0-2.7 \mathrm{~nm}$ and of $2.4-5.2 \mathrm{~nm}$ respectively. The interlamellar spacing between graphene layers is about in the range of 0.34 $-0.35 \mathrm{~nm}$.

HRTEM images of ultrathin sections prepared from T300 carbon fibers are reported in Figure 2, it has been observed that such graphene based films have the relatively loose orientation structures. The stack height $\left(L_{c}\right)$ and the width $\left(L_{a}\right)$ of such graphenes are obviously seen in the rage of $0.8-2.0 \mathrm{~nm}$ and of $2.2-3.1 \mathrm{~nm}$ respectively. The interlamellar spacing between graphene layers is about in the range of $0.34-0.36 \mathrm{~nm}$. After the layer
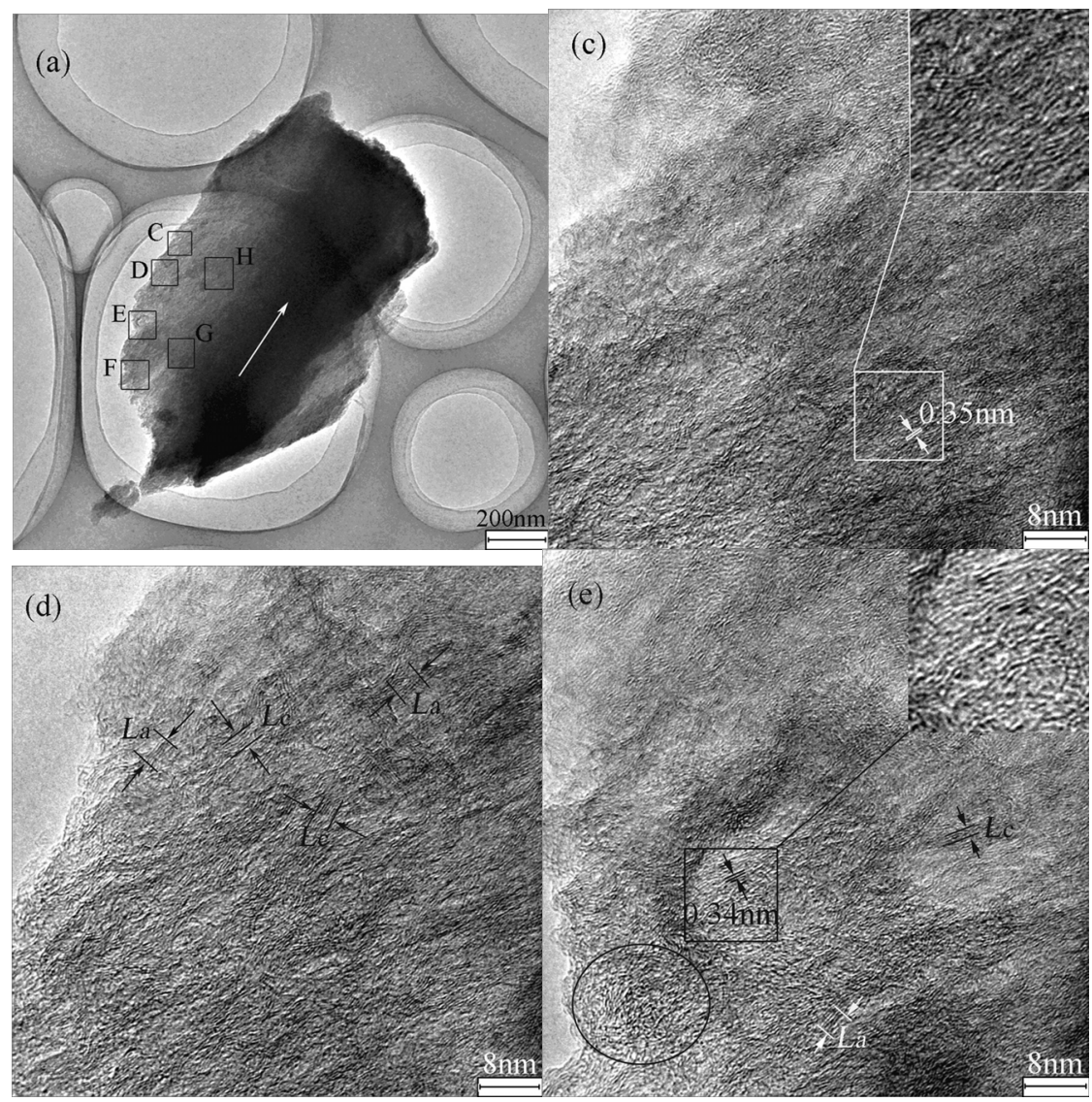

(e)

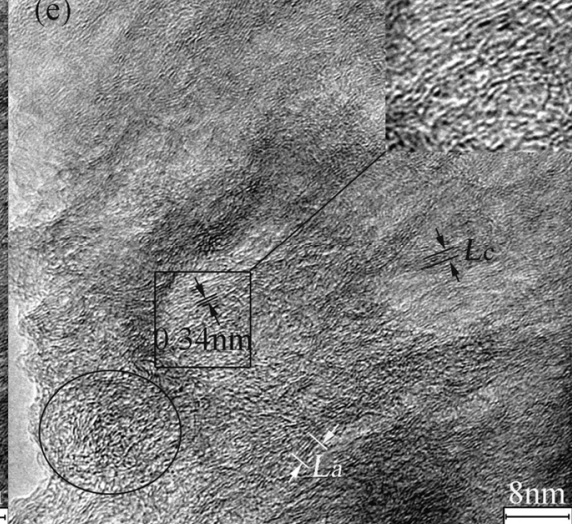

Figure 1. HRTEM images of ultrathin sections prepared from T700 carbon fibers, (a) low magnification image, the direction of white arrow is defined as the direction of axis; (c), (d), (e) is high magnification image of C, D, E areas in Figure1 (a), $L_{c}$ and $L_{a}$ is the stack height and the width in the graphene respectively. 


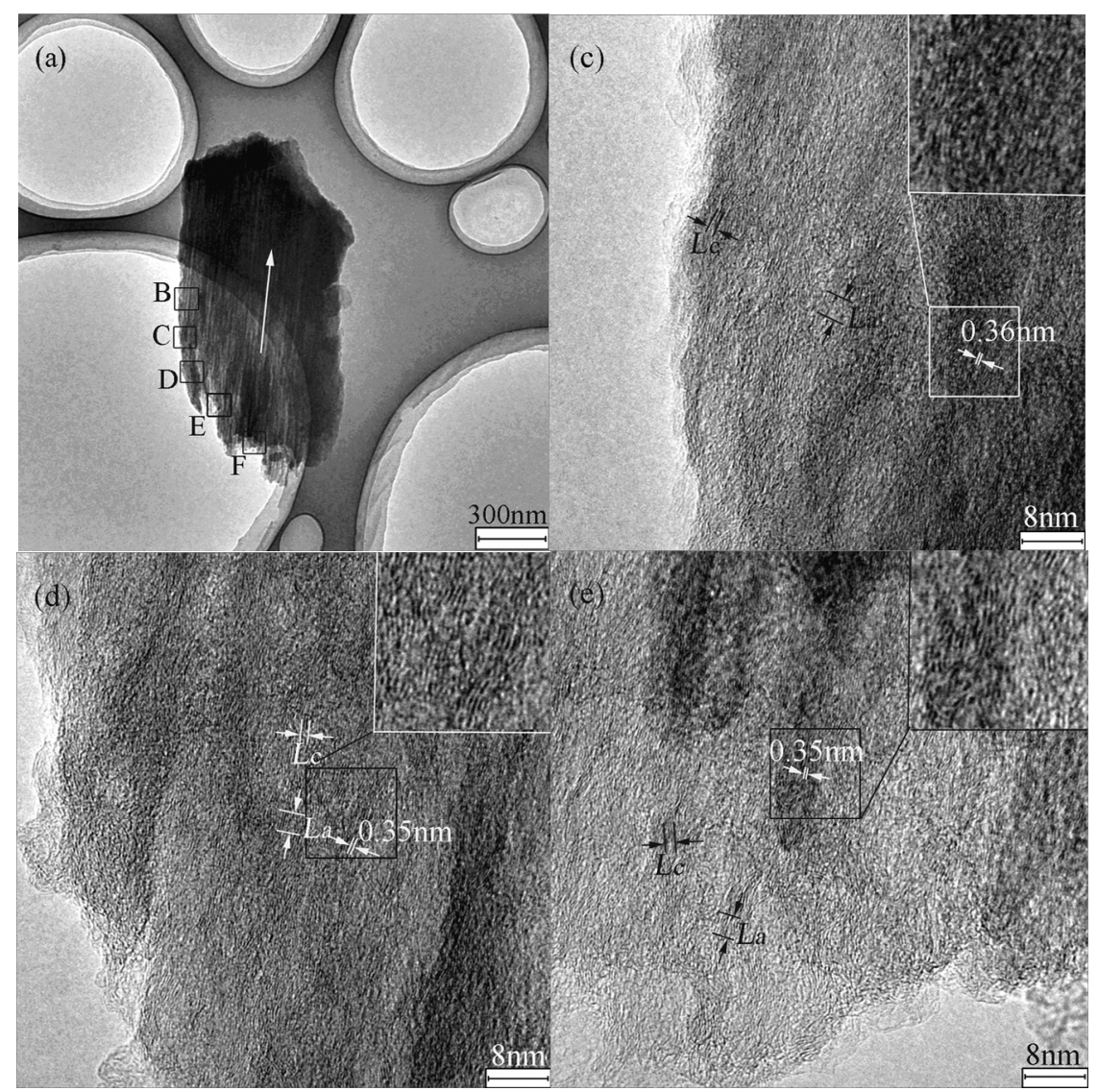

Figure 2. HRTEM images of ultrathin sections prepared from T300 carbon fibers, (a) low magnification image, the direction of white arrow is defined as the direction of axis; (c), (d), (e) is high magnification image of C, D, E areas in Figure 2(a) respectively, $L_{c}$ and $L_{a}$ is the stack height and the width in the graphene respectively.

structure turned up in the square region of Figure2(a) being partially enlarged, it has been found that the length of each graphene layer in such films is about $4 \mathrm{~nm}$ on the average, which testifies the coherence of such graphene layers be worse.

\subsection{Raman Spectrum Analysis of Graphene Based Films}

The Raman spectroscopy of graphene thin films and T300 carbon fibers are shown in Figure 3. It appeared that the common features which are so-called G and D peaks in the $1000-2000 \mathrm{~cm}^{-1}$, the peak at G-band (1580 - $1600 \mathrm{~cm}^{-1}$ ) which belongs to the Raman-allowed phonon mode $E_{2 g}$, which involves out-of-phase displacements, the peak at D peak $\left(1350-1370 \mathrm{~cm}^{-1}\right)$ which is the disorder-induced phonon mode due to the infinite size of crystals and defects. But it has been investigated that a notable peak at $1620 \mathrm{~cm}^{-1}$ appeared for the graphene based thin films, which results from the variation of carbon atoms on the surface of graphene, also, it has been demonstrated that the twist of graphene planeresults in an obvious reduction of distance and the increase of

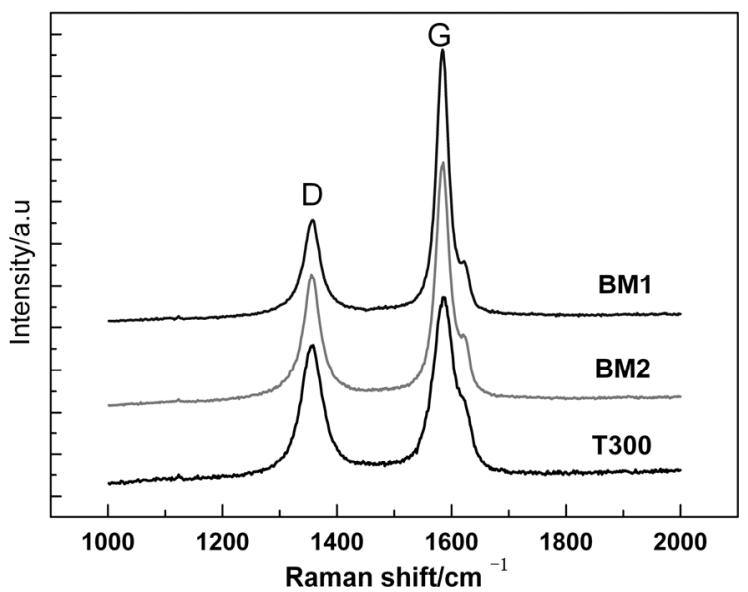

Figure 3. Raman spectroscopy of the graphene based films and T300 carbon fibers, BM1 and BM2 graphene based films were prepared from and T700 and T300 carbon fibers respectively.

mutual action among carbon atoms. The half width of G-band for ultrathin section prepared from T700 carbon fibers is the smaller and becomes comparatively sharp 
when compared with that of ultrathin section prepared from T300 carbon fibers, which suggests the graphene layer be the more parallel along the fiber axis.

The intensity ratio of the D-band against the G-band $\left(R=I_{\mathrm{D}} / I_{\mathrm{G}}\right)$ is widely used to evaluate the quality of graphene materials, it shows the dependence both on the degree of graphitization and the orientation of graphite planed in the surface of graphene materials. As calculated from the data obtained from Figure 3, the intensity ratio of a spectral line $R_{\mathrm{BM} 1}=0.52<R_{\mathrm{BM} 2}=0.65<R_{\mathrm{T} 300}=$ 0.78 , which clearly demonstrates the relative content of $s p^{2}$-hybridized carbon atoms in BM1 films should be the highest when compared with that of BM2, on the other hand, its skeleton structure of carbon atoms becomes more regular, and its lamellar spacing is more complete and compact.

\subsection{XRD Analysis}

The samples were also investigated by XRD as shown in Figure 4, it has been reported that there is the same common evident peak at $2 \theta=25^{\circ}$ which is termed as the (002) reflection, the weak peak at $2 \theta=43^{\circ}$ which is ascribed to (100) reflection, the other weak peak at $2 \theta=$ $55^{\circ}$ which is attributed to (004) reflection. The stack height $\left(L_{c}\right)$ and the planar spacing $\mathrm{d}_{002}$ can be calculated by the Scherrer formula and the Bragg equation respectively. The $\mathrm{d}_{002}$ spacing calculated of $0.332 \mathrm{~nm}$ for the graphene based in BM1 films is slightly smaller than that of $0.345 \mathrm{~nm}$ for BM2 films and that of $0.356 \mathrm{~nm}$ for T300, the stack height $\left(L_{c}\right)$ calculated of $2.05 \mathrm{~nm}$ for the graphene based in BM1 films is larger than that of 1.76 $\mathrm{nm}$ for BM2 films and that of $1.44 \mathrm{~nm}$ for T300. This indicates that the graphene based in BM1 films have relatively high graphitization.

The half-value width in the (002) reflection for BM1 films is smaller than that of BM2 films, which results in the increasing of graphene based crystallite thickness and the reducing of interlayer spacing. So the stacking layers of crystallite become more and more, the porosity among the crystallite is smaller. It has been found that the diffraction peak in the (002) reflection for BM1 films is fairly sharp, the intensity is extremely high, which suggest that the planes of hexatomic ring of carbon orderly stack in the intermolecular acting force, the degree of orientation for such graphene is better than that of BM2 due to its high stereo-tacticity.

\section{Conclusions}

The graphene based films prepared from carbon fibers has been developed by a novel cutting technique -the ultramicrotomy. The distance between the network-graphene plane in graphene based films prepared from T700 carbon fibers decreases, the order design of graphene is

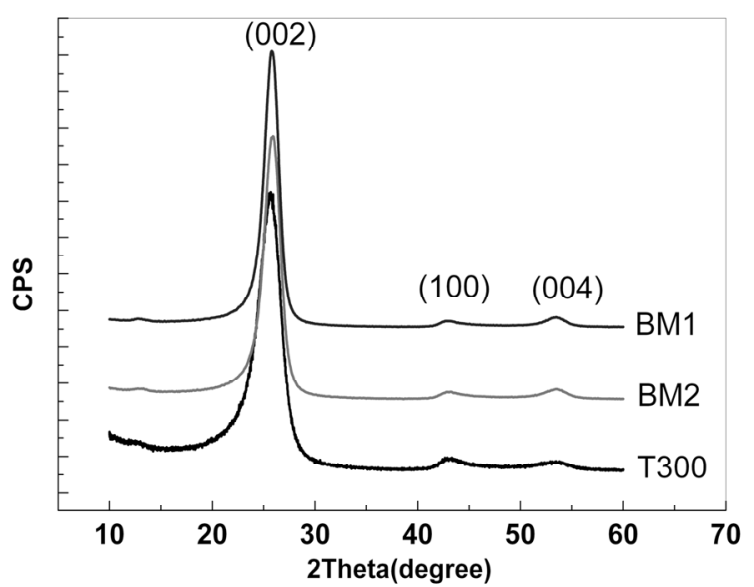

Figure 4. XRD patterns of the graphene based films and T300 carbon fibers, BM1 and BM2 graphene based films were prepared from and T700 and T300 carbon fibers respectively.

denser and the arrangement shows a preferred orientation along the drawing direction. The relative content of the forming $S P^{2}$-hybridized orbit of carbon atoms in the graphene based films prepared from T700 carbon fibers is higher.

\section{Acknowledgements}

The authors are very grateful to Graduate Independent Innovation Foundation of Shandong University, GIIFSDU (2009TS004) and National Basic Research Program of China (2011CB605601) for providing financial support.

\section{REFERENCES}

[1] S. Stankovich, D. A. Dikin, G. H. B. Dommett, K. M. Kohlhaas, E. J. Zimney, E. A. Stach, R. D. Piner, S. T. Nguyen and R. S. Ruoff, "Graphene-Based Composite Materials," Nature, Vol. 442, No. 7100, 2006, pp. 282286. doi:10.1038/nature04969

[2] H. L. Chun, L. Li, F. M. Kin, W. F. George and F. H. Tony, "Ultraflat Grapheme," Nature, Vol. 462, No. 7271, 2009, pp. 339-341. doi:10.1038/nature08569

[3] A. K. Geim, "Graphene: Status and Prospects," Science, Vol. 324, No. 5934, 2009, pp. 1530-1534. doi:10.1126/science. 1158877

[4] J. C. Meyer, A. K. Geim, M. I. Katsnelson, K. S. Novoselov, T. J. Booth and S. Roth, "The Structure of Suspended Graphene Sheets," Nature, Vol. 446, No. 7131, 2007, pp. 60-63. doi:10.1038/nature05545

[5] K. S. Novoselov, A. K. Geim, S. V. Morozov, D. Jiang, Y. Zhang, S. V. Dubonos, I. V. Grigorieva and A. A. Firsov, "Electric Field Effect in Atomically Thin Carbon Films," Science, Vol. 306, No. 5696, 2004, pp. 666-669. doi:10.1126/science. 1102896

[6] T. Kyotani, N. Sonobe and A. Tomita, "Formation of 
Highly Orientated Graphite from Polyacrylonitrile by Using a Two-Dimensional Space between Montmorillonite Lamellae," Nature, Vol. 331, No. 6154, 1988, pp. 331-333. doi:10.1038/331331a0

[7] G. Eda, G. Fanchini and M. Chhowalla. "Large-Area Ultrathin Films of Reduced Graphene Oxide as a Transparent and Flexible Electronic Material," Nature Nanotechnology, Vol. 3, No. 5, 2008, pp. 270-274. doi:10.1038/nnano.2008.83

[8] D. A. Dikin, S. Stankovich, E. J. Zimney, R. D. Piner, G. H. B. Dommett, G. Evmenenko, S. T. Nguyen and R. S. Ruoff, "Preparation and Characterization of Graphene Oxide Paper," Nature, Vol. 448, No. 7152, 2007, pp. 457460. doi:10.1038/nature06016

[9] L. Tapaszto, G. Dobrik, P. Lambin and L. P. Biro, "Tailoring the Atomic Structure of Graphene Nanoribbons by Scanning Tunnelling Microscope Lithography," Nature Nanotechnology, Vol. 3, No. 7, 2008, pp. 397-401. doi:10.1038/nnano.2008.149

[10] C. M. Chen, Q. H. Yang, Y. G. Yang, W. Lv, Y. F. Wen, P. X. Hou, M. Z. Wang and H. M. Cheng, "Self-Assembled Free-Standing Graphite Oxide Membrane," Advanced Materials, Vol. 21, 2009, pp. 3007-3011. doi:10.1002/adma.200990138

[11] L. Y. Jiao, X. R. Wang, G. Diankov, H. L. Wang and H. J. Dai, "Facile Synthesis of High-Quality Graphene Nanoribbons," Nature Nanotechnology, Vol. 5, No. 5, 2010, pp. 321-325. doi:10.1038/nnano.2010.54

[12] L. J. Ci, L. Song, D. Jariwala, A. L. Elı'as, W. Gao, M. Terrones and P. M. Ajayan, "Graphene Shape Control by Multistage Cutting and Transfer," Advanced Materials, Vol. 21, 2009, pp. 4487-4491. doi:10.1002/adma.200900942

[13] C. A. Di, D. C. Wei, G. Yu, Y. Q. Liu, Y. L. Guo and D. B. Zhu, "Patterned Graphene as Source/Drain Electrodes for Bottom Contact Organic Field-Effect Transistors," Advanced Materials, Vol. 20, No. 17, 2008, pp. 32893293. doi:10.1002/adma.200800150

[14] X. L. Li, G. Y. Zhang, X. D. Bai, X. M. Sun, X. R. Wang, E. G. Wang and H. J. Dai, "Highly Conducting Graphene Sheets and Langmuir-Blodgett Films," Nature Nanotechnology, Vol. 3, No. 9, 2008, pp. 538-542. doi:10.1038/nnano.2008.210

[15] L. Y. Jiao, L. Zhang, X. R. Wang, G. Diankov and H. J. Dai, "Narrow Graphene Nanoribbons from Carbon Nano- tubes," Nature, Vol. 458, No. 7240, 2009, pp. 877-880. doi:10.1038/nature07919

[16] G. Y. Zhang, P. F. Qi, X. R. Wang, Y. R. Lu, X. L. Li, R. Tu, S. Bangsaruntip, D. Mann, L. Zhang and H. J. Dai, "Selective Etching of Metallic Carbon Nanotubes by GasPhase Reaction," Science, Vol. 314, 2006, pp. 974-976. doi:10.1126/science.1133781

[17] S. S. Datta, D. R. Strachan, S. M. Khamis and A. T. C. Johnson, "Crystallographic Etching of Few-Layer Graphene," Nano Letter, Vol. 8, 2008, pp. 1912-1915. doi:10.1021/n1080583r

[18] Z. S. Wu, "Efficient Synthesis of Graphene Nanoribbons Sonochemically Cut from Graphene Sheets," Nano Research, Vol. 3, No. 1, 2010, pp. 16-22.

[19] D. V. Kosynkin, "Longitudinal Unzipping of Carbon Nanotubes to form Graphene Nanoribbons," Nature, Vol. 458, No. 7240, 2009, pp. 872-876. doi: $10.1038 /$ nature 07872

[20] C. Lee, X. D. Wei, J. W. Kysar and J. Hone, "Measurement of the Elastic Properties and Intrinsic Strength of Monolayer Graphene," Science, Vol. 321, 2008, pp. 385 389. doi:10.1126/science. 1157996

[21] Y. J. Bai, C. G. Wang, N. Lun, Y. X. Wang, M. J. Yu and B. Zhu, "HRTEM Microstructures of PAN Precursor Fibers," Carbon, Vol. 44, No. 9, 2006, pp. 1773-1778. doi:10.1016/j.carbon.2005.12.041

[22] Y. X. Wang and Q. Wang, "Evaluation of Carbonization Tar in Making High Performance Polyacrylonitrile-Based Carbon Fibers," Journal of Applied Polymer Science, Vol. 104, 2007, pp. 1255-1259. doi:10.1002/app.25754

[23] R. Perret and W. Ruland, "The Microstructure of PANBase Carbon Fibres," Journal of Applied Crystallography, Vol. 3, No. 6, 1970, pp. 525-532. doi: $10.1107 / \mathrm{S} 0021889870006805$

[24] S. C. Bennet and D. J. Johnson, "Electron-Microscope Studies of Structural Heterogeneity in PAN-Based Carbon Fibres," Carbon, Vol. 17, No. 1, 1979, pp. 25-39. doi:10.1016/0008-6223(79)90067-8

[25] H. Rennhofer, D. Loidl, S. Puchegger and H. Peterlik, "Structural Development of PAN-Based Carbon Fibers Studied by in Situ X-Ray Scattering at High Temperatures under Load," Carbon, Vol. 48, No. 3, 2010, pp. 964-971. doi:10.1016/j.carbon.2009.11.012 